\title{
General decay of solution for a porous-elastic system with weak nonlinear dissipation
}

\author{
SEBASTIÃO M. S. CORDEIRO, MAURO L. SANTOS AND CARLOS A. RAPOSO ${ }^{\dagger}$
}

\author{
Date of Receiving : $\quad 14.01 .2018$ \\ Date of Revision : 28.04 .2018 \\ Date of Acceptance : 14.06 .2018
}

\begin{abstract}
In this work the asymptotic behaviour of solution for one-dimensional equations of an homogeneous and isotropic porous elastic solid is analyzed. We use a classic result of P. Martinez [1] to obtain the general decay result. We give some example to illustrate the energy decay rates and consider also the case of the polynomial growth.
\end{abstract}

\section{Introduction}

Elastic solids with voids is one of the simple extensions of the theory of the classical elasticity. It allows the treatment of porous solids in which the matrix material is elastic and the interstices are void of material (see Goodman and Cowin [2] and Nunziato and Cowin [3] for details).

It is worth noting that in the last decades, research on porous elastic systems has been made considering various types of dissipative mechanisms. We describe here some of the main results. To do this, let us consider the following evolution equations in one-dimensional case

$$
\left\{\begin{array}{c}
\rho u_{t t}=T_{x} \\
J \phi_{t t}=H_{x}+G
\end{array}\right.
$$

2010 Mathematics Subject Classification. 35Q70, 35B40, 74F05.

Key words and phrases. Porous-elasticity, exponential decay, localized damping.

The authors thanks the anonymous referees for their valuable comments as well as suggestions that helped to improve the presentation of this paper. The first author has been partially supported by the CNPq Grant 163428/2014-0. The second author has been partially supported by the CNPq Grant 311553/2013-3 and the third author was partially supported by PNPD/UFBA/CAPES(Brazil).

Communicated by: Mauricio S. Cortes and Shiv K. Kaushik

${ }^{\dagger}$ Corresponding author. 